\title{
Simulated properties of printed antennas on silicon substrates for THz/sub-THz arrays
}

\author{
M.V. Sakhno ${ }^{1,2}$, J.V. Gumenjuk-Sichevska ${ }^{1}$, F.F. Sizov ${ }^{{ }^{*}}$ \\ ${ }^{I}$ V. Lashkaryov Institute of Semiconductor Physics, 41, prospect Nauky, 03028 Kyiv, Ukraine, \\ ${ }^{2}$ Taras Shevchenko Kyiv National University, 64, Volodymyrs 'ka str., 01601 Kyiv, Ukraine \\ *Corresponding author e-mail: sizov@isp.kiev.ua
}

\begin{abstract}
Some properties of printed antennas and arrays for uncooled silicon plasmon detector arrays based on field effect transistors are shortly discussed. Antenna geometry has been optimised for maximising gain at $300 \mathrm{GHz}$. It is shown that for bow-tie antennas in the arrays in diffraction limit approximation, there are almost no cross-talks between the neighbour antennas. It is shown that the gain for one antenna is higher than that for antenna, in the array.
\end{abstract}

Keywords: bow-tie antenna, THz, FET detectors, antenna array.

Manuscript received 15.10.10; accepted for publication 02.12.10; published online 28.02.11.

\section{Introduction}

$\mathrm{THz}$ technologies are actively spread now in different areas of human activity. Numerous recent breakthroughs have pushed $\mathrm{THz}$ research into the stage of wide public use. One of the important components of these technologies are detectors [1], and one of the key components of $\mathrm{THz} / \mathrm{sub}-\mathrm{THz}$ devices is an antenna providing transfer of a signal to the electromagnetic field of radiation or, for the reciprocal process, converting electromagnetic field into a signal (voltage or current) in the detector.

The simplest one as well as the best understood is the dipole antenna (see, e.g. [2]). For radiation effective transmission and reception its length should be close to the half of the wavelength $\lambda$. The feature drawbacks important for effective radiation reception by $\mathrm{THz} / \mathrm{sub}$ $\mathrm{THz}$ detectors of this kind of antenna are its low gain, lack of directionality, narrow bandwidth. A large number of much more effective antennas were proposed [2], including dish and horn antennas, log-periodic, spiral, slot/aperture, etc. and antenna arrays based on some of them. But most of them are difficult to fabricate. Bow-tie antenna is one of the best option in ratio performance/difficult of fabrication. Comparison of different IR antennas can be found in [3], and functioning peculiarities are discussed in, e.g., [4].

From the practical point of view, to get low-cost active imaging instruments in $\mathrm{THz} / \mathrm{sub}-\mathrm{THz}$ region, it is important to use uncooled arrays of detectors. Among a number of different detectors proposed and realized for
$\mathrm{THz} /$ sub-THz spectral region in recent two decades, the concept of $\mathrm{THz}$ detection based on plasma-wave excitation in two-dimensional electron gas in field effect transistors (FETs) [5-7] integrated with readout integrated circuits (ROICs), though they are still in the stage of research and optimisation, seems one of the most attractive as makes it possible to develop large scale integrated devices based on conventional large scale integration (LSI) technologies. Here, an attempt to optimize antenna geometry and antenna position in array for field-effect silicon transistors used for uncooled $\mathrm{THz} / \mathrm{sub}-\mathrm{THz}$ detectors is undertaken.

It is important to maximise antenna performance. Correct antenna geometry should be chosen. In this investigation, we optimise bow-tie antenna geometry for maximising gain at the frequency $300 \mathrm{GHz}$ without using dielectric lenses. We also investigate the influence of the substrate thickness and mutual antennas influence.

\section{Antenna modelling}

Antenna's behaviour is similar in receiving and transmitting modes. That's why, the antenna was modelled in the transmitting mode, as this procedure is simpler and more accurate. To provide computation, we chose the method of moments (MoM). Most of all, we were interested in the antenna gain. The gain is proportional to the antenna effective area. The detector sensitivity is proportional to the antenna effective area, too. The relation between the antenna effective area $S_{\text {eff }}$ and antenna gain $G$ is $[2,3]$ 


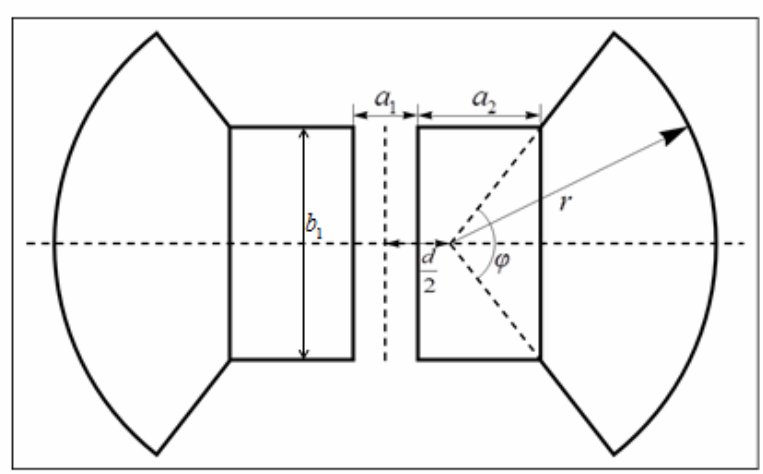

Fig. 1. The antenna geometry.

\begin{tabular}{|c|}
\hline $\mathrm{SiO}_{2}, 285 \mathrm{~nm}$ \\
$A l, 400 \mathrm{~nm}$ \\
\hline$S \mathrm{SiO}, 95 \mathrm{~nm}$ \\
\hline$S t, d_{S i}$ \\
\hline
\end{tabular}

Fig. 2. System cross section.

$S_{\text {eff }}=\frac{\lambda^{2}}{4 \pi} G$,

where $\lambda$ is the wavelength. Thus, with the frequency $v$ increase the effective antenna area is reduced.

Recently new kind of $\mathrm{THz} / \mathrm{sub}-\mathrm{THz}$ detectors was developed, such as silicon plasma field effect transistors (see, e.g. $[6,7]$ ). They can be implemented using highly developed Si technology. The main objective of this work was to optimise antenna geometry for these devices. The bow-tie shape of the antenna was chosen (Fig. 1), because of possible relatively high gain in comparison with other planar printed antennas.

The planar system cross-section is shown in Fig. 2. The middle aluminium layer is an antenna. Passivation layers are located below and above the antenna layer. Silicon dioxide layer is manufactured during the FET production procedure. Silicon is the substrate where FET detectors were formed. At the backside of the substrate, the metallization layer is formed.

As optimisation parameters of the antenna, we used $a_{2}, r, d_{S i}, \varphi$, see Figs.1, 2. Aluminium has high electric conductance in this frequency range, that's why it can be modelled as an ideal conductor. The antenna geometric parameters and materials electric constants are listed in Table 1 and Table 2, respectively.

A lot of peaks (Fig. 3) appearing in the gain dependences on radiation frequency $v$ can be explained by interference of incident wave and wave reflected from metallization layer. The substrate thickness determines interferential maximum or minimum. So, to obtain high gain it is important to choose the proper substrate thickness.
Table 1. Geometrical parameters of antenna.

\begin{tabular}{|c|c|c|c|c|c|c|}
\hline $\begin{array}{c}a_{1}, \\
\mu \mathrm{m}\end{array}$ & $\begin{array}{c}a_{2}, \\
\mu \mathrm{m}\end{array}$ & $\begin{array}{c}r, \\
\mu \mathrm{m}\end{array}$ & $\begin{array}{c}d, \\
\mu \mathrm{m}\end{array}$ & $\begin{array}{c}d_{\mathrm{Si}}, \\
\mu \mathrm{m}\end{array}$ & $\begin{array}{c}\varphi, \\
\mathrm{deg}\end{array}$ & $b_{1}$ \\
\hline 10 & 75.8 & 164 & 20 & 505.5 & 104 & $b_{1}=\left(b_{1}+2 a_{2}-d\right) \times$ \\
& & & & & & $\times \tan \left(\frac{\varphi}{2}\right)$ \\
\hline
\end{tabular}

Table 2. Materials electrical properties.

\begin{tabular}{|l|l|l|}
\hline Material & $\varepsilon$ & $\sigma, S / m$ \\
\hline $\mathrm{Si}$ & 11.7 & 5 \\
\hline $\mathrm{SiO}_{2}$ & 3.9 & 0 \\
\hline
\end{tabular}

Antenna input impedance is not higher than $200 \Omega$ (Fig. 4), so it can be difficult to match it with FET input impedance. In FET detectors, the antennas are coupled to the source and gate inputs. In $\mathrm{THz} / \mathrm{sub}-\mathrm{THz}$ frequency range the impedance of this circuit is much less as compared to the channel one $\left(\sim 10^{7}-10^{10} \Omega\right)$ that generates the output signal and serves for its registration (or is attached to the ROIC input circuit).

Interaction between antennas was analyzed for the system of two antennas with two different antennas relative positions. One of them is for the case when antennas axes are parallel, and the other one is for the case when antennas are arranged along one axis.

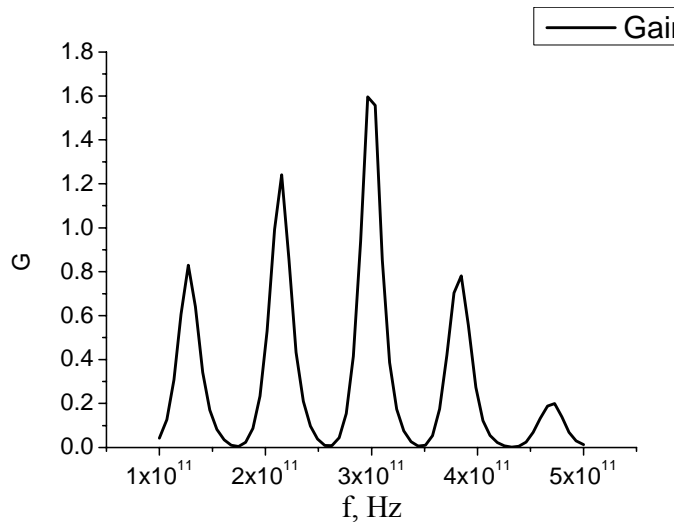

Fig. 3. Gain frequency dependence.

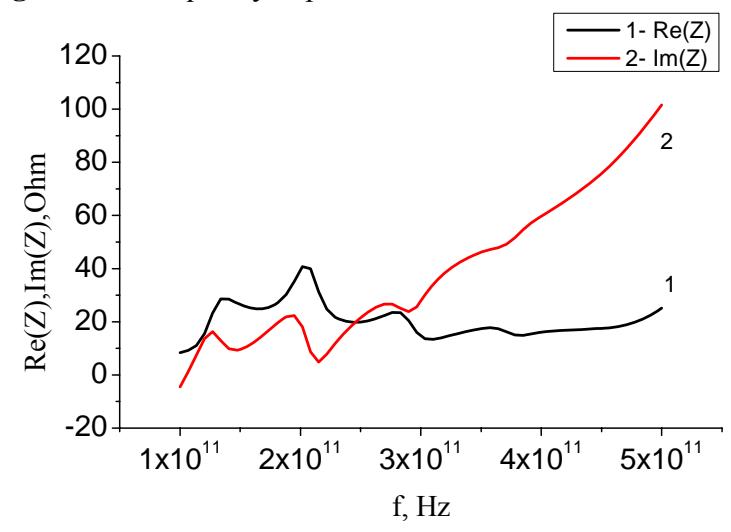

Fig. 4. Antenna input impedance. 


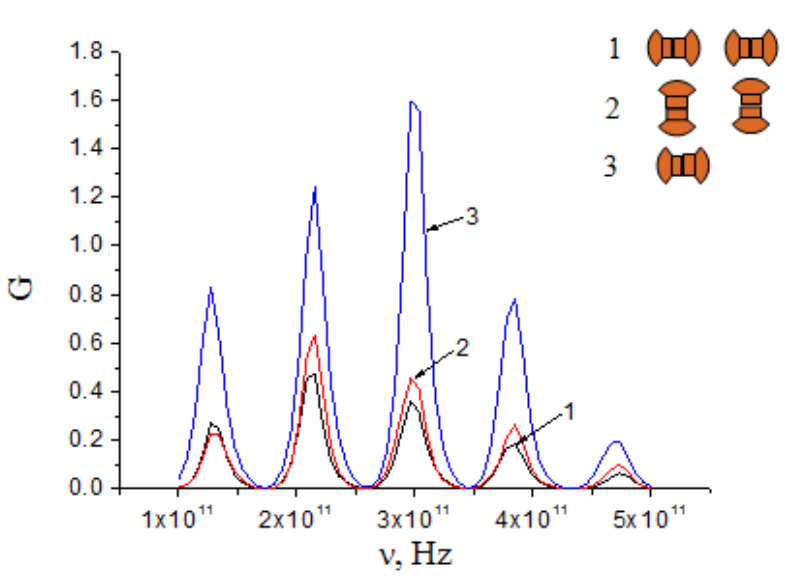

Fig. 5. Gain for one antenna and for two antenna system.

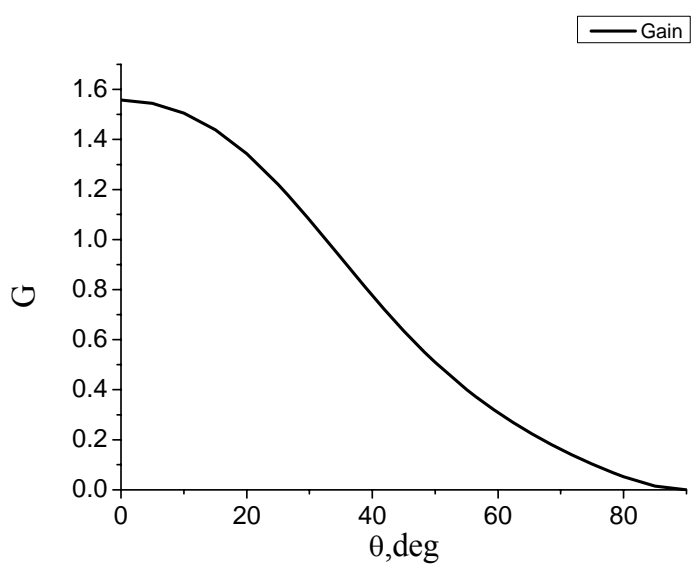

Fig. 6. Antenna gain angular dependence.

It was shown (Fig. 5) that the antennas gain is lower for the two-antenna system in comparison with the single antenna. It can be explained in the following way. Let us imagine that antenna operates in the transmitting mode. The gain is related to the angular dependence of this antenna (Fig. 6). The narrower the angular dependence, the higher the gain is. When there are two antennas, the angular dependence becomes broader. The gain is smaller in this case.

Thus, interaction between antennas exists. It can be described by the "cross-talk" parameter $S_{21}$. The value of $\left|\mathrm{S}_{21}\right|^{2}$ shows an approximate amount of energy which is transferred from the first antenna to another one.

One can see (Fig. 7) that when the distance between antennas is smaller than $900 \mu \mathrm{m}$ interaction is smaller for parallel axes positioning. This pattern should be used in antenna arrays with a short distance between antennas. This interaction is minimal at the distance close to $800 \mu \mathrm{m}$ (Fig. 8).

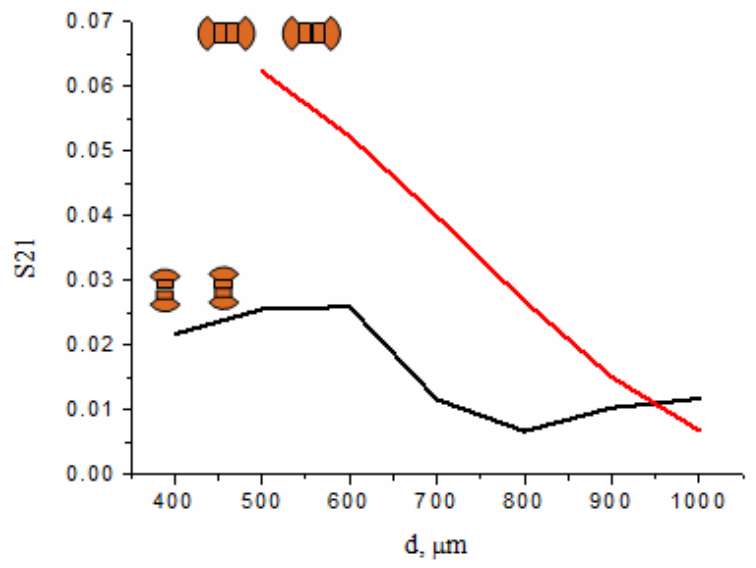

Fig. 7. Interaction of antennas for different system configurations.

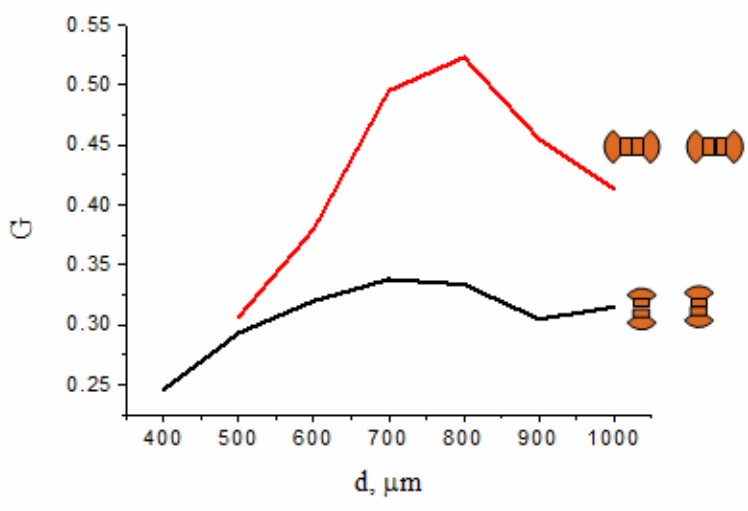

Fig. 8. Gain dependence on distance at the signal frequency $300 \mathrm{GHz}$.

\section{Conclusions}

Some properties of printed antennas and arrays for uncooled silicon plasmon detector arrays based on field effect transistors were investigated. Planar bow-tie shaped antenna geometry has been optimised for maximising the gain at the frequency $300 \mathrm{GHz}$. The antenna has a relatively high gain in comparison with other planar antennas for the $\mathrm{THz}$ range frequencies. Bow-tie antenna + detector system performance depends on the substrate thickness. It has been shown that the gain for the single antenna is larger than the gain for interacting antennas in the array. The power exchange between two antennas is relatively small, but it is sufficient to decrease the gain of the entire system. The optimal distance between detectors was found, which minimized the interaction between neighboring antennas. Therefore, the multielement antenna-detector array of THz-range should be optimized not only on the parameters of each antenna, but, taking into account their mutual influence, on the distance between them and their mutual arrangement. 
1. F. Sizov and A. Rogalski, THz detectors // Progress in Quantum Electronics 34(5), p. 278-347 (2010).

2. C. Balanis, Antenna Theory: Analysis and Design. John Wiley \& Sons, Inc, Hoboken (2005).

3. F.J. González and G.D. Boreman, Comparison of dipole, bowtie, spiral and log-periodic IR antennas // Infrared Physics \& Technology 46(5), p. 418-428 (2005).

4. D.B. Rutledge, S.E. Schwarz and A.T. Adams, Infrared and submillimetre antennas // Infrared Physics, 18(5-6), p. 713-729 (1978).

5. W. Knap, V. Kachorovskii, Y. Deng, S. Rumyantsev, J.Q. Lü, R. Gaska, M.S. Shur, G. Simin, X. Hu, M.A. Khan, C.A. Saylor and L.C. Brunel, Nonresonant detection of terahertz radiation in field effect transistors // J. Appl. Phys. 91(11), 9346 (2002).
6. E. Öjefors, A. Lisauskas, D. Glaab, H. Roskos and U. Pfeiffer, Terahertz imaging detectors in CMOS technology // J. Infrared, Millimeter and Terahertz Waves, 30(12), p. 1269-1280 (2009).

7. W. Knap, M. Dyakonov, D. Coquillat, F. Teppe, N. Dyakonova, J. Łusakowski, K. Karpierz, M. Sakowicz, G. Valusis, D. Seliuta, I. Kasalynas, A. El Fatimy, Y. Meziani and T. Otsuji, Field Effect Transistors for Terahertz Detection: Physics and First Imaging Applications // J. Infrared, Millimeter and Terahertz Waves, 30(12), p. 13191337 (2009). 